\title{
HANDWRITTEN SCRIPT AND WORD RECOGNITION - A VIEW-BASED APPROACH
}

\author{
Marek Tabedzki, Khalid Saeed \\ Faculty of Computer Science, Bialystok Technical University \\ Wiejska 45A, 15-351 Bialystok, Poland \\ \{abedzki, aida\}@ii.pb.bialystok.pl, http://aragorn.pb.bialystok.pl/ zspinfo/
}

\begin{abstract}
This paper presents a hybrid system for character and word recognition. It is based on a modification to the view-based approach presented in authors' previous works. The algorithm is appropriate for dealing with whole, unsegmented words or isolated characters. The characteristic vectors taken from views of the tested image are processed with the method of minimal eigenvalues of Töeplitz matrices. The obtained series of minimal eigenvalues are used for classification with Artificial Neural Networks. The results of the experiments on different sets of words and letters are presented.
\end{abstract}

Keywords: Character recognition, word recognition without segmentation, minimal eigenvalues of Töeplitz matrices, neural networks.

\section{INTRODUCTION}

The main concept of this method is based on a hybrid approach using the idea of fusing the viewbased algorithm with the algorithm of minimal eigenvalues of Töeplitz matrices. The essential ideas of the view-based recognition system were presented in [1].

This method is based on the fact that for correct image recognition we usually need only partial information about its shape - its silhouette or contour. Thus, the idea is to analyze only the top and bottom views of a word or character. The view is a set of pixels belonging to a contour of an image having extreme values of one of its coordinates. It describes the general shape of the analyzed image, hence being good base for further classification.

At first [2-3], this method was used for recognizing separate Latin characters (both printed and handwritten). The obtained results were good and promising. Using only the top and bottom views was problematic because some characters (e.g. "E" and "Z") could not be distinguished in such a way. Thus, the left and right views were introduced - they were necessary in recognizing some letters. Not all pixels of views were analyzed, but rather only a small number of the characteristic points. The vectors of these points were the base for further analysis with the method of minimal eigenvalues of Töeplitz matrices while the classification was considered with Artificial Neural Networks.
In next works [4-6] this method was used for recognizing unsegmented words. This approach bypasses the stage of segmentation - the whole word is treated as a single image, and processed in that way. In case of words only two views - top and bottom - are analyzed.

View-Based approach allows us to obtain good description of the tested image, which can be used for direct classification. But our evaluation shows, that method of Töeplitz matrices (introduced as another stage of analysis) can further improve the results.

The classification can be done with any appropriate method. In early works we used the method of simple comparison - classical $k$-Nearest Neighbor classifier. In our researches, the method of Töeplitz matrices proved to give very good results and high Success Rate when used together with an ANN-based classifier. Hence, we also used Artificial Neural Networks [7] as classifiers in our performed experiments.

For the tests we gathered database of words and characters. In case of characters we used both machine-printed and handwritten letters of different styles and writers. In case of words, we used printed whole words. Handwritten text, is left to our future work. 


\section{VIEW-BASED METHOD IN A HYBRID APPROACH}

This method is based on the fact, that for the correct shape recognition a human usually needs only partial information about it, simply its silhouette.

We examine number of "views" of the tested image, extracting from them a characteristic vector, which describes the given figure. The view is a set of points that plot one of projections of the object (top or bottom in case of words, and also left or right for letters) - it consists of pixels belonging to the contour of an image and having extreme values of one coordinate (Fig. 1.) - e.g. maximal $y$ coordinate for a given $x$ coordinate in case of top view.

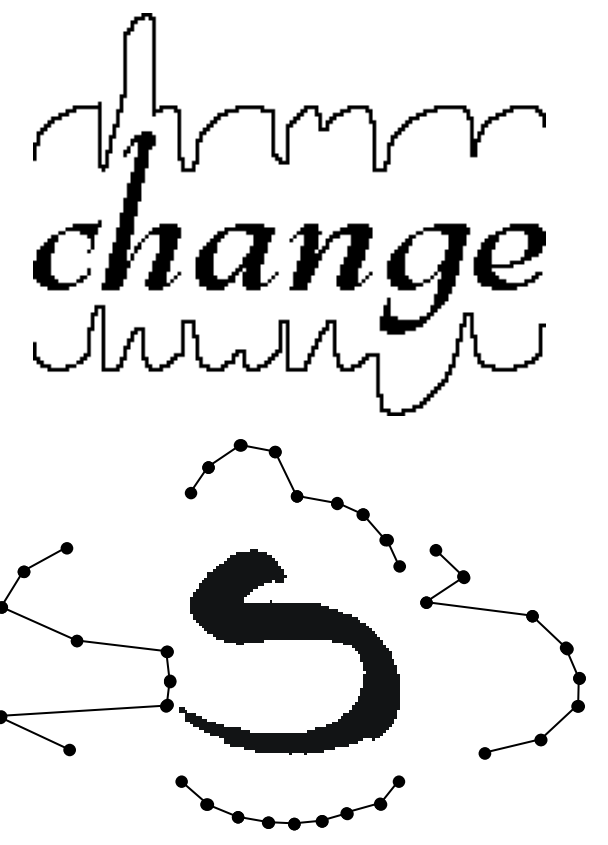

Fig. 1 - Two views of the word "change" and four views of the character " $\mathrm{S}$ ".

It should be noted, that while only two views were used in case of words, for character recognition we used all four views of an image. Another important feature is the unnecessity of the conventional stage of thinning. Only the shape of the word is analyzed, not its skeleton. The only necessary preprocessing stage is the image binarization, to convert the scanned image into a black-and-white one.

Next, characteristic points are marked out on the surface of each view to describe the shape of that view. The number of characteristic points is constant for each analyzed shape, independent of the width or height of the image. This number is fixed for each experiment. The method of selecting the characteristic points may vary. In early works characteristic points were uniformly selected along each view. In our recent works, however, the method of computing them was changed. To find the characteristic points of top and bottom views, one needs to divide the image representing the given word vertically into a number of identical segments equal to the number of points we want to obtain. Next, we find the position of the highest and the lowest pixel in each segment - they are the points of top and bottom views (Fig. 2). Points for left and right views can be obtained analogically.

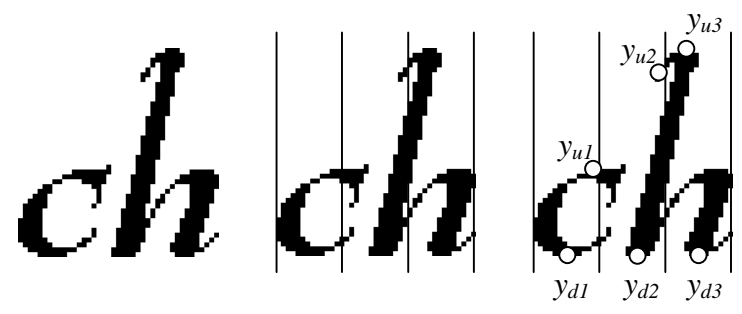

Fig. 2 - Characteristic points of a fragment from the word "change".

The next step is to evaluate the coordinates of the taken points ( $y$ for the top and bottom, $x$ for the left and right views). Thus we obtain $n$-element characteristic vectors describing the given image. The introduced novelty here is the addition of supplementary vector (in case of word recognition) that describes the aspect ratio of the tested image. It consists of two values - width and height of the image.

These obtained vectors describe the given word, and are the base for further analysis. It is also possible to directly use these vectors in the classification process. To stress the characteristics of the tested image, we use an additional transformation stage.

\section{TÖEPLITZ MATRICES}

In the next step the values from the characteristic vectors are treated by the image-describing algorithm supported by the minimal eigenvalues of Töeplitz matrices. Values from each of the obtained views are processed individually, so we create separate series of Töeplitz matrices, and obtain separate series of its minimal eigenvalues for the each analyzed view.

This method has proved its feasibility in many of our previous works. It gave good results for many issues related to recognition of text, voice, signature, etc. However, it is the first time it is applied to whole words.

According to the algorithm described in detail in [8], the values of the normalized vectors are considered as the coefficients of Taylor's series:

$T(p)=c_{0}+c_{1} p+c_{2} p^{2}+\ldots+c_{n} p^{n}+\ldots$

which are used to form Töeplitz forms (Eq.2). 


$$
[H]=\left[\begin{array}{cccc}
c_{0} & c_{1} & c_{2} & \ldots \\
C_{-1} & c_{0} & c_{1} & \ldots \\
C_{-2} & c_{-1} & c_{0} & \ldots \\
\cdots & \ldots & \ldots & \ldots
\end{array}\right]
$$

\section{CLASSIFICATION WITH NEAREST NEIGHBOR METHOD}

For the sake of classification these separate vectors describing views and aspect ratio of the tested word were combined into one vector.

When classifying with the method of simple comparison one needs to gather a set of examples subjects of comparison and the base for classification. These examples are already classified words - vectors calculated in the same manner, as described above, each labeled with the name (or ID number) of its class. This set of examples is the Learning Set. In our experiments we use part (usually 20-30\%) of the collected samples as a Learning Set. The remaining samples of the database are used for test purposes forming the Test Set.

To classify a given word we need to compare its characteristic vector point-to-point to the characteristic vectors of all examples contained in Learning Set, and find the most similar vector - the nearest neighbor of the tested vector. It is the vector to which the distance is minimal. For that calculation we use 1-norm distance (Manhattan distance, Eq.4).

$$
d=\sum_{i=1}^{n}\left|x_{i}-y_{i}\right|
$$

We assume that the tested vector is of the same class as its nearest neighbor, i.e. image in question shows the same word as image described by the vector found in Learning Set.

Now, the nearest neighbor method is in fact the conventional and well known $k \mathrm{NN}$ ( $k$-Nearest Neighbors) approach [10], but with $k=1$. Of course we can use higher $k$ value and expect better results. But, if we want to keep rather a small number of examples for each class, high $k$ value would be inappropriate - searching 5 nearest neighbors within a set of over 100 classes, each represented by 10 examples will not necessary lead to better results.

It is worth considering that with this method of classification we need to compare each tested word with each example contained in Learning Set. There can be a lot of time-consuming calculations, so it is very important to keep the Learning Set as small as possible. Therefore, our efforts are to reduce the number of examples of each class. More experiments with even smaller Learning Set were done, but we are still exploring that field, and further results will be presented in future works. In some cases, the number of examples for a given class can be reduced to about 10 without affecting the efficiency. computations to a minimum. Of course in case need high precision and do not care about the computing time, we may even use all of them. 


\section{CLASSIFICATION WITH ARTIFICIAL NEURAL NETWORKS}

In some cases the method of Nearest Neighbors is quite inconvenient, because it needs to maintain big database of already classified vectors, and in order to recognize a word all the vectors from the database are compared with the vector describing the tested word. In case of huge database it may be timeconsuming and hence inefficient. The use of Artificial Neural Networks allows us to avoid those costs.

In our research we use Multi-Layered Perceptron: classic feed-forward neural network, with one hidden layer, trained by the backpropagation method [7]. As a transfer function, we have taken the bipolar logistic sigmoid function (Eq. 5).

$$
F(n)=\frac{2}{1+e^{-2 n}}-1
$$

The number of inputs to the Neural Network was equal to the total number of the elements in characteristic vectors describing the input image. The number of outputs was equal to the number of classes in our database. The number of neurons in hidden layer was determined according to Kolmogorov's Theorem [11]. First, network was trained with vectors from the Training Set. The training stage was performed until recognition rate on Training Set climbs to $95 \%$. Next, fully trained network was tested with remaining words (words from the Test Set) - the vector describing the tested word (obtained from view-based algorithm) was presented to the input of the applied network. In the output we get information about the class of the input vector (i.e. the word it may describe) - the output neuron on which we observe the biggest signal indicates the number of the classes.

\section{EXPERIMENTS AND RESULTS}

Here we present essential results of our experiments with words and characters. All of presented results are taken from our previous papers [2-6]. Here we select the most interesting and significant ones.

As a first example, we considered the word "SYSTEM" printed with 66 different font styles [2]. Then the word was segmented (this can be achieved with one of known segmentation algorithms [1213]), and separate letters were classified and recognized with our view-based approach - using the method of Töeplitz matrices as another stage of analysis while the Artificial Neural Network as a classifier.

The results have shown a correctness percentage of about $92 \%$, as an arithmetic average. However, the percentage of correctly recognized words (words with each of 6 letters correctly recognized) was calculated to be a bit lower $-73 \%$.

Table 1 presents the results showing the effectiveness of the algorithm and its recognition rate for tested characters.

Table 1. Results for the case of minimal eigenvalues with neural networks

\begin{tabular}{|c|c|c|c|c|c|c|}
\hline & & & & & E & \\
character & correct & S & Y & T & M \\
\hline S & $96.21 \%$ & - & $0.76 \%$ & $0.76 \%$ & $2.27 \%$ & $0.00 \%$ \\
\hline $\mathbf{Y}$ & $87.88 \%$ & $3.03 \%$ & - & $6.82 \%$ & $0.00 \%$ & $2.27 \%$ \\
\hline T & $88.64 \%$ & $6.06 \%$ & $1.52 \%$ & - & $2.27 \%$ & $1.52 \%$ \\
\hline $\mathbf{E}$ & $91.67 \%$ & $6.82 \%$ & $1.52 \%$ & $0.00 \%$ & - & $0.00 \%$ \\
\hline $\mathbf{M}$ & $96.97 \%$ & $1.52 \%$ & $0.00 \%$ & $0.00 \%$ & $1.52 \%$ & - \\
\hline average & $\mathbf{9 2 . 2 7} \%$ & & & & & \\
\hline
\end{tabular}

The table shows that the script $\mathrm{S}$ is recognized correctly (as S) in over 96\%, whereas incorrectly in $2.27 \%$ as $\mathrm{E}$, in $0.76 \%$ as $\mathrm{T}$, and so forth. As can be seen, shapes of most frequently mistaken characters are in fact very similar: "Y" recognized as "T", or "E" recognized as "S" - and therefore, they can easily be classified inaccurately.

For the sake of comparison we have also done some experiments using other approaches. In the next example, characters were classified using sole Neural Network, without eigenvalues of Töeplitz matrices calculation. This time values from characteristic vectors describing four views, were put on the network input. Table 2 presents the obtained results.

Table 2. Recognition results for Neural Networks only

\begin{tabular}{|c|c|c|c|c|c|c|}
\hline character & correct & $\mathbf{S}$ & $\mathbf{Y}$ & $\mathbf{T}$ & $\mathbf{E}$ & $\mathbf{M}$ \\
\hline $\mathbf{S}$ & $95.96 \%$ & - & $1.52 \%$ & $0.00 \%$ & $1.52 \%$ & $1.01 \%$ \\
\hline $\mathbf{Y}$ & $92.93 \%$ & $0.00 \%$ & - & $1.52 \%$ & $1.52 \%$ & $4.04 \%$ \\
\hline $\mathbf{T}$ & $80.81 \%$ & $0.00 \%$ & $13.13 \%$ & - & $4.04 \%$ & $2.02 \%$ \\
\hline $\mathbf{E}$ & $87.88 \%$ & $10.61 \%$ & $0.00 \%$ & $0.51 \%$ & - & $1.01 \%$ \\
\hline $\mathbf{M}$ & $97.47 \%$ & $0.51 \%$ & $0.51 \%$ & $0.00 \%$ & $1.52 \%$ & - \\
\hline average & $\mathbf{9 1 . 0 1 \%}$ & & & & & \\
\hline
\end{tabular}

As we can see the attained effectiveness is only slightly lower than above. When considering word recognition, then the result is only $69 \%$. This means that our method of minimal eigenvalues allows us to achieve better results and higher efficiency.

Another attempt was to classify with classical nearest neighbor method - on the base of minimal Manhattan distance of vectors formed from the series of minimal eigenvalues of Töeplitz matrices. The learning set makes the base, with which the 
characters from the test set are compared. As can be seen, the rate of recognition is not as high as in the method with neural networks - it approximately reaches the rate of $85 \%$ for the correctly recognized characters.

Table 3. Results of recognition for classical methods of classification

\begin{tabular}{|c|c|c|c|c|c|c|}
\hline character & correct & S & Y & T & E & M \\
\hline S & $83.64 \%$ & - & $0.00 \%$ & $1.82 \%$ & $9.09 \%$ & $5.45 \%$ \\
\hline $\mathbf{Y}$ & $92.73 \%$ & $0.00 \%$ & - & $7.27 \%$ & $0.00 \%$ & $0.00 \%$ \\
\hline T & $81.82 \%$ & $1.82 \%$ & $14.55 \%$ & - & $1.82 \%$ & $0.00 \%$ \\
\hline $\mathbf{E}$ & $74.55 \%$ & $18.18 \%$ & $0.00 \%$ & $1.82 \%$ & - & $5.45 \%$ \\
\hline $\mathbf{M}$ & $96.36 \%$ & $0.00 \%$ & $0.00 \%$ & $0.00 \%$ & $3.64 \%$ & - \\
\hline average & $\mathbf{8 5 . 8 2 \%}$ & & & & & \\
\hline
\end{tabular}

Some experiments with the handwritten word "SYSTEM" have been done. The results have shown a percentage of about $80 \%$ of correctly recognized and classified characters. But more investigations with handwritten scripts allowed for better results.

In the same attempt, as with the word "SYSTEM", the word "SOFTWARE" was taken [3] - handwritten 125 times by different people and ways. Similarly, the word was segmented and separate letters were classified and recognized, basing on view-based characteristic vector analyzed with Töeplitz matrices method and using Artificial Neural Networks as a classifier.

Due to some tweaks in the algorithm (e.g. increasing number of computed eigenvalues and altering neural network architecture) the attained effectiveness was greater than that in the former example reaching $87 \%$ of correctly recognized letters. Table 4 presents the recognition results showing the effectiveness of the hybrid algorithm for tested characters.

The table shows that the script $\mathrm{S}$ is recognized correctly (as S) in almost $97 \%$, whereas incorrectly in $1.41 \%$ as $\mathrm{O}$ and in $0.47 \%$ as $\mathrm{F}$, and so forth. The highlighted values are the correctly recognized scripts within this group of letters in one of the letter tested class. As mentioned before, the class considered in this work is that of the letters from the word SOFTWARE.

For comparison, Table 5 shows the results of other experiments. This time classification was done with method of simple comparison (nearest neighbor method) - on the base of vectors formed from the series of minimal eigenvalues of Töeplitz matrices. As can be seen, the rate of recognition reaches approximately $70 \%$ for the correctly recognized characters - it is not as high as in the method with neural networks.

Further experiments were performed on unsegmented words. Two separate databases were used - a set of 75 English names of animals [4-5] and set of 150 most common English words [6]. This list was composed basing on appearance frequency in real-world documents [14]. Each word was printed with 130 to 150 different fonts. As mentioned above, for word recognition only two views are acquired, and only two series of minimal eigenvalues are calculated.

Table 4. Recognition results for the handwritten characters by the hybrid algorithm

\begin{tabular}{|c|r|r|r|r|r|r|r|c|}
\hline character & S & O & F & T & W & A & R & E \\
\hline S & $96.71 \%$ & $1.41 \%$ & $0.47 \%$ & $0.24 \%$ & $0.00 \%$ & $0.00 \%$ & $0.47 \%$ & $0.71 \%$ \\
\hline $\mathbf{O}$ & $1.65 \%$ & $90.59 \%$ & $0.24 \%$ & $0.00 \%$ & $0.47 \%$ & $0.00 \%$ & $2.59 \%$ & $4.47 \%$ \\
\hline $\mathbf{F}$ & $0.00 \%$ & $0.00 \%$ & $88.00 \%$ & $2.12 \%$ & $0.94 \%$ & $0.94 \%$ & $4.71 \%$ & $3.29 \%$ \\
\hline $\mathbf{T}$ & $0.00 \%$ & $0.00 \%$ & $3.06 \%$ & $95.53 \%$ & $0.71 \%$ & $0.24 \%$ & $0.47 \%$ & $0.00 \%$ \\
\hline $\mathbf{W}$ & $1.65 \%$ & $2.59 \%$ & $1.88 \%$ & $2.59 \%$ & $87.53 \%$ & $1.18 \%$ & $1.65 \%$ & $0.94 \%$ \\
\hline $\mathbf{A}$ & $3.06 \%$ & $1.18 \%$ & $0.71 \%$ & $0.00 \%$ & $1.88 \%$ & $89.88 \%$ & $3.29 \%$ & $0.00 \%$ \\
\hline $\mathbf{R}$ & $2.12 \%$ & $2.35 \%$ & $6.12 \%$ & $3.29 \%$ & $1.41 \%$ & $3.06 \%$ & $74.82 \%$ & $6.82 \%$ \\
\hline $\mathbf{E}$ & $3.29 \%$ & $4.00 \%$ & $7.53 \%$ & $0.00 \%$ & $0.94 \%$ & $0.47 \%$ & $9.18 \%$ & $74.59 \%$ \\
\hline
\end{tabular}

Table 5. Handwritten characters classified with classical method

\begin{tabular}{|c|r|r|r|r|r|r|r|r|}
\hline character & S & O & F & T & W & A & R & E \\
\hline S & $83.00 \%$ & $10.00 \%$ & $0.00 \%$ & $0.00 \%$ & $1.00 \%$ & $1.00 \%$ & $5.00 \%$ & $0.00 \%$ \\
\hline $\mathbf{O}$ & $1.00 \%$ & $92.00 \%$ & $0.00 \%$ & $0.00 \%$ & $0.00 \%$ & $2.00 \%$ & $3.00 \%$ & $2.00 \%$ \\
\hline $\mathbf{F}$ & $0.00 \%$ & $3.00 \%$ & $54.00 \%$ & $7.00 \%$ & $4.00 \%$ & $6.00 \%$ & $6.00 \%$ & $20.00 \%$ \\
\hline $\mathbf{T}$ & $0.00 \%$ & $0.00 \%$ & $10.00 \%$ & $89.00 \%$ & $0.00 \%$ & $0.00 \%$ & $0.00 \%$ & $1.00 \%$ \\
\hline $\mathbf{W}$ & $4.00 \%$ & $7.00 \%$ & $15.00 \%$ & $1.00 \%$ & $50.00 \%$ & $4.00 \%$ & $3.00 \%$ & $16.00 \%$ \\
\hline $\mathbf{A}$ & $2.00 \%$ & $2.00 \%$ & $0.00 \%$ & $0.00 \%$ & $0.00 \%$ & $93.00 \%$ & $2.00 \%$ & $1.00 \%$ \\
\hline $\mathbf{R}$ & $2.00 \%$ & $12.00 \%$ & $7.00 \%$ & $4.00 \%$ & $3.00 \%$ & $9.00 \%$ & $56.00 \%$ & $7.00 \%$ \\
\hline $\mathbf{E}$ & $3.00 \%$ & $11.00 \%$ & $8.00 \%$ & $0.00 \%$ & $1.00 \%$ & $7.00 \%$ & $20.00 \%$ & $50.00 \%$ \\
\hline
\end{tabular}


Words from the animal set were classified using the method of the nearest neighbor. This method allows us to achieve $80 \%$ correctly recognized words. Table 6 presents detailed results for some of the tested words.

Table 6. Results of recognition for selected words from the animal set

\begin{tabular}{|c|c|}
\hline Animal Name & Recognition Rate \\
\hline monkey & $98 \%$ \\
\hline dog & $95 \%$ \\
\hline donkey & $89 \%$ \\
\hline parrot & $84 \%$ \\
\hline rabbit & $76 \%$ \\
\hline mule & $48 \%$ \\
\hline horse & $42 \%$ \\
\hline
\end{tabular}

As can be seen the best result was obtained for the word "monkey" - $98 \%$ of tested samples were correctly recognized. The worst result was in the case of the word "horse" with only $42 \%$ recognition rate. All in all, the results are promising - for over one-half of the tested words the recognition rate is greater than $80 \%$.

Table 7 presents the most common mistakes. It shows the wrongly recognized words and how often they occur wrongly. The first column contains tested words, the second shows the false result and the third the percentage of the miss case to the whole number of iterations.

Table 7. Most common mistakes for Animal Set

\begin{tabular}{|c|c|c|}
\hline Word & $\begin{array}{c}\text { Recognized } \\
\text { as }\end{array}$ & Rate \\
\hline Mule & mole & $32 \%$ \\
\hline Horse & heron & $29 \%$ \\
\hline skunk & shark & $23 \%$ \\
\hline Shark & skunk & $21 \%$ \\
\hline heron & horse & $20 \%$ \\
\hline Coral & camel & $20 \%$ \\
\hline
\end{tabular}

For example, the word "mule" was in $32 \%$ recognized as "mole". However, and from the other side as can easily be seen, the shapes of mistaken words are too similar: "heron" and "horse", "mule" and "mole", "skunk" and "shark" - and therefore they can easily be classified inaccurately. One of the solutions to that problem is to add some characteristic points, and gather more data to distinguish them more accurately. But, because we want to avoid large characteristic vectors and hence long computations, we would rather add another stage in the system of recognition, to set apart words of similar shapes. This will be the subject of our future works.

Samples from the set of most common English words were classified using Artificial Neural Networks. This method of classification has ended with $82 \%$ correctly recognized words. Once more, method of Töeplitz matrices has proved to work better in the hybrid system with neural-based classifiers. Table 8 presents the detailed results for some selected words

Table 8. Results of recognition for selected words

\begin{tabular}{|c|c|}
\hline Word & Recognition Rate \\
\hline change & $100 \%$ \\
\hline might & $96 \%$ \\
\hline night & $91 \%$ \\
\hline make & $85 \%$ \\
\hline head & $76 \%$ \\
\hline house & $59 \%$ \\
\hline from & $54 \%$ \\
\hline
\end{tabular}

As can be seen, all tested samples of the word "change" were correctly recognized. On the other hand, for the word "from" the recognition rate was only $54 \%$. On the whole the results are promising for over one-half of the tested words, the recognition rate does not drop below $80 \%$.

Table 9. Most common mistakes

\begin{tabular}{|c|c|c|}
\hline Word & Recognized as & Rate \\
\hline cover & never & $17 \%$ \\
\hline house & home & $13 \%$ \\
\hline at & of & $12 \%$ \\
\hline down & draw & $11 \%$ \\
\hline head & hand & $11 \%$ \\
\hline hand & head & $10 \%$ \\
\hline
\end{tabular}

Table 9 presents some of the miss cases. And again, shapes of mistaken words are very similar: "house" and "home", "hand" and "head", "down" and "draw" leading to the high ratio of false recognition.

It should be mentioned here, however, that some fonts (mainly calligraphic or decorative) have lowered the success rate of the recognition. In fact, with more than half of the used fonts, the obtained recognition rate was over $85 \%$ and for some of them the effectiveness was even nearly $100 \%$. The problem with untypical fonts can be solved by further expansion of the Learning Set. 


\section{CONCLUSION}

The hybrid method presented in this paper combines View-Based method with the method of minimal eigenvalues of Töeplitz matrices and Artificial Neural Networks as a classifier. The hybrid system of Töeplitz matrices and ANN had already proved its high recognition rate in spoken word and speaker recognition [15].

The results of the experiments have shown that this method is significantly efficient for Latin script and printed-word recognition. Although the performance and the efficiency for some of the words are not so high, generally the results are promising and encouraging for further work. The problem of miss classification can be resolved by adding another stage to the system of recognition dealing with problematic pairs of words. Alternatively, the stage of classification can be extended into tree-like process, with separate classes in its leaves. The presented method is elastic and easily accepts further improvements and adjustments. The future work will therefore concentrate on a more effective way of obtaining characteristic values. We would also perform more experiments with handwritten words.

Our work has continuously been compared with other authors' results. De Oliveira et al. [16] used Hidden Markov Models [17] classifiers for recognition of month names, obtaining recognition rate of $75.9 \%$. The same author applied $\mathrm{NN}$ classifiers on the same base of words to achieve $81.8 \%$ recognition rate. Larger database was used by Lavrenko et al. in his work concerning handwritten words recognition in historical documents [18] Recognizing words without segmentation was in a $65 \%$ classifying rate. Compared with these achievements, our results are promising and good indeed.

Of course the obtained results may not always be directly comparable, because of the different data sets used.

\section{ACKNOWLEDGEMENTS}

This work is an extended version to the paper entitled "View-Based Word Recognition System" presented at $6^{\text {th }}$ ICCNAI'2006 conducted in Brest, Belarus.

The work is supported by the Rector of Bialystok Technical University, grant no. W/WI/3/04.

\section{REFERENCES}

[1] K. Saeed. M. Tabędzki. M. Adamski. A New Approach for Object-Feature Extract and Recognition. 9th International Conference on Advanced Computer Systems - ACS'02.
Miedzyzdroje, 23-25 October 2002. pp. 389397.

[2] K. Saeed, M. Tabedzki. Hybrid System for Letter Recognition. 3rd International Conference on Neural Networks and Artificial Intelligence - ICNNAI'03. Minsk, 2003. pp. 4448.

[3] K. Saeed, M. Tabedzki. A New Hybrid System for Recognition of Handwritten-Script. COMPUTING - International Scientific Journal of Computing. Institute of Computer Information Technologies, Volume 3, Issue 1, pp. 50-57, Ternopil 2004.

[4] M. Tabedzki, K. Saeed. View-Based Word Recognition System. Proc. 4th Intern. Conf. on Neural Networks and Artificial Intelligence ICNNAI'06. Brest, Belarus, 2006, pp. 145-148

[5] M. Tabedzki, K. Saeed. A View-Based Töeplitz-Matrix-Supported System for Word Recognition without Segmentation. Proc. 6th Intern. Conf. on Intelligent System Design and Applications - ISDA'06. Jinan, Shandong, China, 2006. (Best Paper Award)

[6] M. Tabedzki, K. Saeed. Hybrid Word Recognition System. Proc. 13th Intern. MultiConf. on Advanced Computer Systems ACS-AIBITS/CISIM'06. Miedzyzdroje, Poland, 2006.

[7] S. Haykin. Neural Networks. A Comprehensive Foundation. Prentice Hall, New Jersey, USA, second edition, 1999.

[8] K. Saeed. On The Realization of Digital Filters. Proc. The 1st International Conference on Digital Signal Processing and its Applications DSPA'98. Vol. 1, Moscow, 1998, pp. 141-143.

[9] K. Saeed. A New Approach in Image Classification. Proc. 5th International Conference on Digital Signal Processing and its Applications - DSPA'03. Moscow 2003. Vol. 1, pp. 49-52.

[10]G. Shakhnarovish, T. Darrell, and P. Indyk, Nearest-Neighbor Methods in Learning and Vision. The MIT Press, 2005.

[11]A. N. Kolmogorov. On the Representation of Continuous Functions of Several Variables by Superposition of Continuous Functions of One Variable and Addition. Dokl. Akad. Nauk SSSR, 1957, vol. 114, pp. 953-956.

[12]R. C. Gonzalez and R. E Woods. Digital Image Processing. Addison-Wesley Publishing Company. 1992. pp. 7-9, 413-414.

[13]K. Bowyer and N. Ahuja. Advances in Image Understanding. IEEE Computer Society Press, 1996. p. 65.

[14]http://www.world-english.org

[15]K. Saeed, M. Nammous. A Simple Speech-andSpeaker Identification System. Accepted for 
publication in IEEE Trans. on Ind. Electronics Humatronics. New York, USA, 2006.

[16]J.J. De Oliveira Junior, J.M. De Carvalho, C.O.D.A Freitas, R. Sabourin. Evaluating NN and HMM Classifiers for Handwritten Word Recognition. Computer Graphics and Image Processing, Elect. Engg Dept., Fed. Univ. of Campina Grande, Brazil, 2002, pp. 210-217.

[17]D. Guillevic, C.Y. Suen, HMM Word Recognition Engine. Fourth International Conference on Document Analysis and Recognition (ICDAR97). 1997, pp. 544-547.

[18]V. Lavrenko, T.M. Rath, R. Manmatha, Holistic word recognition for handwritten historical documents. Document Image Analysis for Libraries. 2004, pp. 278-287.

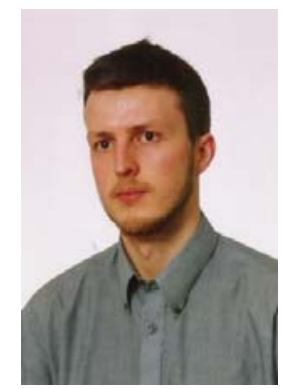

Marek Tabedzki received his M.Sc. degree (distinction) in Computer Science from Bialystok Technical University (Poland) in 2001. He is presently with Bialystok Technical University at Faculty of Computer Science. His research interests include Information Processing Systems, particularly Digital Image Processing and Pattern Recognition.

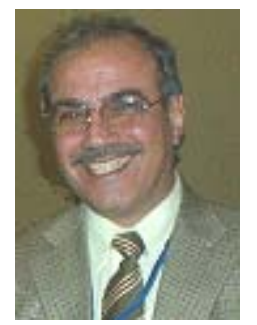

Khalid Saeed received his B.SC. degree in Electrical Engineering from University of Baghdad in 1976, his M.Sc. degree in Electronics in 1978 and Ph.D. (distinction) in Telecommunications in 1981. Both of his M.Sc. and Ph.D. he received from Wroclaw University of Technology. He is presently with Bialystok Technical University at Department of Computer Engineering (head 1994-2000), Faculty of Computer Science (Vice-Dean 1996-1999). His research interests include Information Processing Systems and their Hybrid Algorithms and applications in Image Analysis and Description. 